\title{
PACKING AND COVERING A TREE BY SUBTREES
}

\author{
I. BÁRÁNY, J. EDMONDS and L. A. WOLSEY
}

Received 27 September 1984

For two polyhedra associated witl packing subtrees of a tree, the structure of the vertices is described, and efficient algorithms are given for optimisation over the polyhedra. For the related problem of covering a tree by subtrees, a reduction to a packing problem, and an efficient algorithm are presented when the family of trees is "fork-free".

\section{Introduction}

Given a tree $R$ with vertex set $V$ and a family of its subtrees $\mathscr{F}$, consider the problem of packing these subtrees into the tree:

$$
\begin{array}{cc} 
& \operatorname{Max} \sum\{c(T) x(T): T \in \mathscr{F}\} \\
\text { subject to } & \sum\{x(T): T \ni v\} \leqq 1 \\
& x(T) \in\{0,1\} \quad(T \in V), \\
&
\end{array}
$$

where $c(T)$ is a weight function on $\mathscr{F}$.

It is known that this problem can be solved by a polynomial-time algorithm as the intersection graph of $\mathscr{F}$ is triangulated [3] and the maximum weighted stable set problem on a triangulated graph can be solved efficiently [2], see also [4].

In this paper we consider various generalisations of problem $P_{0}$. For related problems involving all subtrees of a tree, see [5].

In order to state our results we need some preparation. First we assume that the tree is rooted (with root $r \in V$ ). This induces a partial ordering of $V$ in the usual way: we say that $u<v(u, v \in V)$ if $u \neq v$ and $u$ belongs to the unique path connecting $r$ and $v$. We are also given a monotone non-decreasing real valued function $a: V \rightarrow \mathbf{R}$, i.e., $u, v \in V, u<v$ implies $a(u) \leqq a(v)$.

The first problem we consider is the linear program

$$
\operatorname{Max} \sum\{c(T) x(T): T \in \mathscr{F}\}: x \in Q_{1}
$$

where $Q_{1} \subset R_{+}^{|F|}$ is described by the inequalities:

$$
\begin{aligned}
\sum\{x(T): T \ni v\} & \leqq a(v) \quad(v \in V), \\
x(T) & \geqq 0 \quad(T \in \mathscr{F}) .
\end{aligned}
$$

AMS subject classification (1980): 68 E 10, 05 C 05, 05 B 40 
We describe a dual greedy algorithm for $P_{1}$, which can also be viewed as a dynamic programming algorithm. This is one way to obtain a characterisation of the vertices of $Q_{1}$, which are integer whenever $a: V \rightarrow \mathbf{R}$ is an integer valued monotone nondecreasing function.

The second problem we consider is also a packing problem, except that the family $\mathscr{F}$ of subtrees is replaced by the family of all subtrees, and a somewhat special objective function. We consider the linear program:

$$
\operatorname{Max} \sum_{u, v \in V} c(u, v) x(u, v): x \in Q_{2}
$$

where $Q_{2} \subseteq \mathbf{R}^{||^{*}}$ is described by the inequalities

$$
\begin{aligned}
\sum_{u \in V} x(u, v) & \leqq a(v) \quad(v \in V), \\
x(u, v)-x(u, w) & \leqq 0 \quad \text { if } \quad w \in[u, v] \quad(u, v \in V) \\
x(u, v) & \geqq 0 \quad(u, v \in V),
\end{aligned}
$$

where there is a variable $x(u, v)$ for each pair $u, v \in V$, and where $[u, v]$ denotes the vertices on the path connecting $u$ and $v$.

Take a solution $x(u, v)$ to $P_{2}$. Fix $u$ and set $\alpha_{0}=\min \{x(u, v): v \in V, x(u, v)>0\}$ and $S_{0}^{u}=\{v \in V: x(u, v)>0\}$. If $S_{0}^{u} \neq \emptyset$, then $S_{0}^{u}$ is a tree containing $u$. Now define inductively $S_{i}^{u}=\left\{v \in V: x(u, v)>\alpha_{i-1}\right\}$ and $\alpha_{i}=\min \left\{x(u, v): x(u, v)>\alpha_{i-1}\right\}$ unless $S_{i}^{u}=\emptyset$. Clearly $S_{i}^{u}$ is a tree containing $u$ and $x(u, v)=\alpha_{0} s_{0}^{u}(v)+\left(\alpha_{1}-\alpha_{0}\right)$ $s_{1}^{u}(v)+\left(\alpha_{2}-\alpha_{1}\right) s_{2}^{u}(v)+\ldots$ where $s_{i}^{u}$ is the characteristic function of $S_{i}^{u}$, i.e., $s_{i}^{u}(v)=1$ if $v \in S_{i}^{u}$ and 0 otherwise. So we may think of a solution $x(u, v)$ of $P_{2}$ as a weighted sum of subtrees $S_{i}^{u}$ of $R$, each $S_{i}^{u}$ being "rooted" at $u$. Relative to problem $\left(P_{1}\right)$, here $\mathscr{F}$ is the set of all subtrees but the objective value associated with a subtree $T$ is

$$
\max _{u \in T}\left\{\sum c(u, v): v \in T\right\} .
$$

Here again we obtain a characterisation of the vertices of $Q_{2}$, and a dual greedy algorithm to solve the linear program. The problem $P_{2}$ with $a(v)=1$ for all $v \in V$ was the major motivation for this work, as it generalises the tree packing problems considered in [1].

The third problem we consider is that of covering the tree $R$ by subtrees from $\mathscr{F}$. This can be formulated as the integer program:

$$
\begin{aligned}
& \min \sum\{c(T) x(T): T \in \mathscr{F}\} \\
& \sum\{x(T): T \ni v\} \geqq 1 \quad(v \in \mathcal{V}) \\
& x(T) \in\{0,1\} \quad(T \in \mathscr{F}) .
\end{aligned}
$$

While $C_{0}$ is generally NP-hard, we show that when the family $\mathscr{F}$ of subtrees has a certain property, denoted forkfree, which generalises a property of distance subtrees, problem $C_{0}$ can be reduced to problem $P_{2}$ and hence solved efficiently.

We mentioned already that $P_{0}$ is a maximum weighted stable set problem on a triangulated graph. It is shown in Section 5 that the two problems are equivalent.

It is known that when the constraint matrix appearing in $C_{0}$ is totally balanced, $\mathrm{C}_{0}$ can be solved by linear programming. What is the relationship between 
totally balanced matrices and node versus subtree incidence matrices when the subtree family is forkfree? This and similar questions are discussed in Section 5. In addition we return to $P_{2}$ via the problem that was the starting point of this research, the economic lot sizing problem with backlogging.

\section{Packing with a given family of subtrees}

Some notation is needed. A subtree and its set of vertices are denoted by the same letter. For any subtree $T$ of $R$ we call $r(T)=\operatorname{Min}\{v: v \in T\}$ the root of $T$. This is clearly well-defined. For $v \in V$ we denote $R(v)$ the subtree spanned by the vertices $\{u \in V: u \geqq v\}$. Note that if $u<v$, then $[u, v]=\{w \in V: u \leqq w \leqq v\}$. The predecessor $p(v)$ of node $v$ is the first vertex on the path going from $v$ to $r$. The successor set $S(v)$ of vertex $v$ is the set of vertices $w \in V$ having $v$ as a predecessor. The successor set $S(T)$ of a tree $T$ is the set of vertices $w \notin T$ with $p(w) \in T$.

We assume that $a(v) \geqq 0$ for each $v \in V$, as otherwise $Q_{1}$ and $Q_{2}$ are empty. Finally, for $v \in V$ we write $\mathscr{F}(v)=\{T \in \mathscr{F}: r(T)=v\}$.

Here we consider the problem $\mathrm{P}_{1}$, and the associated polytope $Q_{1}$. We shall also need the linear progranming dual of $P_{\mathbf{1}}$ :

$$
\begin{gathered}
\min \sum_{v \in V} a(v) y(v) \\
\sum\{y(v): v \in T\} \geqq c(T) \quad(T \in \mathscr{F}) \\
y(v) \geqq 0 \quad(v \in V) .
\end{gathered}
$$

First we describe an algorithm for $D_{1}$.

The dual greedy algorithm for $\mathrm{D}_{1}$

All vertices are initially unmarked. Set $d(T)-c(T)$ for $T \in \mathscr{F}$.

i) Choose a vertex $v \in V$ that is unmarked but all $w \in R(v) \backslash\{v\}$ are marked.

ii) Fix dual variables

Set $y(v) \leftarrow \max \{0, \max \{d(T): T \in \mathscr{F}(v)\}\}$

$$
=\left\{\begin{array}{l}
0 \text { if } \mathscr{F}(v)=\emptyset \text { or } d(T) \leqq 0 \text { for all } T \in \mathscr{F}(v) \\
d\left(T_{v}\right) \text { for some } T_{v} \in \mathscr{F}(v) \text { otherwise. }
\end{array}\right.
$$

Mark $v$. For later use we fix $T_{v} \in \mathscr{F}(v)$ with $y(v)=d\left(T_{v}\right)$ if $y(v)>0$, ties are broken arbitrarily.

iii) Update $d(T), T \in \mathscr{F}$.

Set $d(T) \leftarrow d(T)$ if $T \in \mathscr{F}, v \notin T$.

Set $d(T) \leftarrow d(T)-y(v)$ if $T \in \mathscr{F}, v \in T$.

iv) If $r$ is unmarked, return to i).

Otherwise, stop.

Observe that the vector $y$ constructed this way does not depend on the dual objective function, $a(v), v \in V$. Note also that on termination of the algorithm $y$ is dual feasible and $d(T) \leqq 0$ for each $T \in \mathscr{F}$. These facts follow by an easy induction argument. 
The greedy algorithm for $\mathbf{P}_{1}$ algorithm.

Here we make use of the subtrees $T_{v}$ that were fixed during the dual greedy

All vertices are initially unmarked. Set $b(v) \leftarrow a(v)$ for $v \in V$.

i) Choose a vertex $v \in V$ that is

a) unmarked

b) for which there is no unmarked vertex $w<v$.

ii) Fix primal variables.

If $y(v)=0$, set $x(T)=0$ for all $T \in \mathscr{F}(v)$.

If $y(v)>0$, set $x\left(T_{v}\right)=b(v), x(T)=0$ for $T \in \mathscr{F}(v) \backslash T_{v}$.

Mark vertex $v$.

iii) Update $b(u), u \in V$.

If $y(v)=0, b$ is unchanged.

If $y(v)>0$, set $b(u) \leftarrow b(u)-x\left(T_{v}\right), u \in T_{v}$.

$$
b(u) \leftarrow b(u), \quad \text { otherwise. }
$$

iv) If all nodes are marked, stop.

If not, return to i).

Theorem 1. If $y \in \mathbf{R}_{+}^{|V|}$ and $x \in \mathbf{R}_{+}^{|F|}$ are constructed by the dual greedy and the greedy algorithm respectively, $y$ is optimal in $\mathrm{D}_{1}$ and $x$ is optimal in $\mathrm{P}_{1}$.

Proof. It is easily seen that $y$ and $x$ are dual and primal feasible as $d(T) \leqq 0, T \in \mathscr{F}$, and $b(u) \geqq 0, u \in V$ respectively. From step iii) of the greedy algorithm for $\mathrm{P}_{\mathbf{1}}$, we see that $y(v)>0$ only if $b(v)=0$. Also $x(T)>0$ only if $d(T)=0$. Hence complementary slackness holds and both solutions are optimal.

An alternative view of the algorithm used in solving $P_{1}$ and $D_{1}$ will be useful later. Let $G(v)$ be the optimal value of $\mathrm{P}_{1}$ when $a(u)=0$ for all $u \in V \backslash R(v), a(u)=1$ for all $u \in R(v)$. The associated solutions are called 1-packings of $R(v)$. In other words $G(v)$ is the optimal value of a 1-packing of $R$ when restricted to the trees $T \in \mathscr{F}$ that lie in $R(v)$.

$$
\begin{gathered}
\text { A recursion for } \mathrm{P}_{1} \\
G(v)=\operatorname{Max}\left\{\sum_{w \in S(v)} G(w), \operatorname{Max}_{T \in \mathscr{F}(v)}\left(c(T)+\sum_{w \in S(T)} G(w)\right)\right\} .
\end{gathered}
$$

This says that there are essentially two possibilities. Either the optimal solution contains no tree $T \in \mathscr{F}$ including the vertex $v$. In this case the solution must consist of optimal 1-packings of each of the trees $R(w), w \in S(v)$. Alternatively if some tree $T$ containing $v$ is used in the optimal solution, the remainder of the solution must consist of optimal 1-packings of the trees $R(w), w \in S(T)$.

The connection between the recursive algorithm and the dual greedy algorithm is readily seen by observing, that

$$
y(v)=G(v)-\sum_{w \in S(v)} G(w)
$$


satisfies

$$
y(v)=\operatorname{Max}\left\{0, \operatorname{Max}_{T \in \mathscr{F}(w)}\left(c(T)-\sum_{w \in T \backslash\{v\}} y(w)\right)\right\}
$$

which coincides with the dual variables given by the dual greedy algorithm.

Now let $x^{v}$ denote an optimal 1-packing of $R(v)$ with value $G(v)$.

Claim. Let

$$
x=\sum_{v \in V} x^{v}(a(v)-a(p(v)))
$$

where $a(p(r))=0$ by definition, and $y$ be defined by $(1)$, then $x \in \mathbf{R}_{+}^{|\vec{F}|}$ and $y \in \mathbf{R}_{+}^{|\boldsymbol{r}|}$ are optimal solutions to $\mathrm{P}_{1}$ and $\mathrm{D}_{1}$ respectively.

Proof. Clearly $x \geqq 0$ as $x^{v}(T) \geqq 0$ and $a(v) \geqq a(p(v))$ for $v \in V$. To see that $x$ satisfies $\sum\{x(T): T \ni w\} \leqq a(w)$, note that $\sum\left\{x^{v}(T): T \ni w\right\} \leqq 1$ and $\sum\left\{x^{v}(T): T \ni w\right\}=$ $=0$ if $v \neq w$ and hence

$$
\begin{aligned}
\sum\left\{\sum_{v \in V} x^{v}(T)(a(v)-a(p(v))): T \ni w\right\} & =\sum_{v \leqq w} \sum_{T \ni w} x^{v}(T)(a(v)-a(p(v))) \leqq \\
& \leqq \sum_{v \leqq w}[a(v)-a(p(v))] \leqq a(w) .
\end{aligned}
$$

Its value is $\sum_{v £ V} G(v)(a(v)-a(p(v)))$.

To show its optimality note that $y(v)$ is a dual feasible solution of value

$$
\sum_{v \in V} a(v) y(v)=\sum_{v \in V} a(v)\left(G(v)-\sum_{w \in S(v)} G(w)\right)=\sum_{v \in V} G(v)(a(v)-a(p(v))) .
$$

We have now shown

Theorem 2. Every vertex of $Q_{1}$ is of the form

$$
x=\sum_{v \in V} x^{v}(a(v)-a(p(v)))
$$

\section{Packing with all possible subtrees}

Here we consider problem $\mathrm{P}_{2}$. The approach is similar to that of the previous section. We use a dual greedy or recursive algorithm to calculate the optimal value $H(v)$ of a packing for $\mathrm{P}_{2}$ when $a(u)=1$ for $u \in R(v)$ and $a(u)=0$ otherwise, and let $x^{v} \in \mathbf{R}^{|V|^{2}}$ be the associated optimal 1-packing. The family of 1-packings $x^{v}, v \in V$ is then used to construct an optimal packing for $\mathrm{P}_{2}$ for any $a: V \rightarrow R^{1}$ that is nondecreasing outwards from $r$.

\section{$A$ recursive algorithm for $\mathrm{P}_{2}$}

In order to calculate $H(v)$ recursively starting from the leaves and working inward towards the root, we use an auxiliary function $\varphi_{u}(v)$ which is essentially the optimal value of a 1-packing of $R(v)$ with the extra condition $x(u, v)=1$. But these is another point of view in looking at $\varphi_{u}(v)$ that will be useful. Remember that the tree $R$ is rooted at $r$ but, as we mentioned in the introduction, it is conve- 
nient to think of a solution $x(u, v)$ of $\mathrm{P}_{2}$ as a weighted sum of subtrees $S_{i}^{u}$ "rooted" at node $u$. If the solution $x(u, v)$ takes $0-1$ values only (which will be the case with the 1-packing of $R(v)$ ), then where is at most one subtree $S^{u}$ rooted at $u$ and $S^{u} \cap$ $\cap S^{v}=0$ for $u \neq v$. In this case we say that the subtree $S^{u}$ covers the node $v$ provided $x(u, v)=1$. More precisely, we define $\varphi_{u}(v)$ as follows: If $u \geqq v, \varphi_{u}(v)$ is the optimal weight of a 1-packing of $R(v)$ where $v$ is covered by a subtree $S^{u}$ rooted at $u$. If $u \neq v$, then $\varphi_{u}(v)$ is the optimal weight of a 1 -packing of $R(v) \cup[u, v]$ where $v$ is covered by a subtree $S^{u}$ rooted at $u$, and only the values of covering vertices of $R(v)$ are counted. The formal definition is this:

$$
\text { if } \begin{aligned}
u \geqq v, \varphi_{u}(v)= & \max \sum_{w \in R(v)} \sum_{z \in R(v)} c(w, z) x(w, z): x \in Q_{2}, \\
& x(u, v)=1, x(w, z)=0 \text { if } w \notin R(v) \text { or if } z \notin R(v) \\
& x(w, z) \in\{0,1\} \quad \text { otherwise } \\
\text { if } u \neq v, \varphi_{u}(v)= & \max \sum_{w \in R(v) \cup(u)=\in R(v)} c(w, z) x(w, z): x \in Q_{2}, \\
& x(u, v)=1, x(w, z)=0 \text { if } w \notin R(v) \cup\{u\} \text { or } z \notin R(v) \\
& x(w, z) \in\{0,1\} \quad \text { otherwise. }
\end{aligned}
$$

Now we obtain:

$$
H(v)=\operatorname{Max}\left\{\sum_{w \in S(v)} H(w), \operatorname{Max}_{u \in R(v)} \varphi_{w}(v)\right\}
$$

because in an optimal 1-packing of $R(v)$ either no subtree covers vertex $v$, or vertex $v$ is covered by a subtree rooted at $u$ where $u \in R(v)$.

The recursion for $\varphi_{u}(v)$ is given by:

$$
\begin{gathered}
\varphi_{u}(v)=c(u, v)+\sum_{w \in S(v)} \operatorname{Max}\left\{\varphi_{u}(w), H(w)\right\} \quad \text { if } \quad u \notin R(v) \backslash\{v\}, \\
\varphi_{u}(v)=c(u, v)+\sum_{w \in S(v) \backslash\left\{w^{\prime}\right\}} \operatorname{Max}\left\{\varphi_{u}(w), H(w)\right\}+\varphi_{u}\left(w^{\prime}\right) \text { if } u \in R(v) \backslash\{v\}
\end{gathered}
$$

where $w^{\prime}=S(v) \cap[u, v]$ is the first vertex after $v$ on the path $[v, u]$.

To justify this recursion, we decompose the value of the 1-packing of $R(v)$ into $c(u, v)$ plus the value of the packing of the trees $R(w), w \in S(v)$. When $u \notin$ $\notin R(v) \backslash\{v\}$, the value of the packing of $R(w)$ is $\varphi_{u}(w)$ if $x(u, w)=1$, and $H(w)$ if $x(u, w)=0, w \in S(v)$. When $u \in R(v) \backslash\{v\}$, the argument is the same except that $x(u, v)=1$ implies $x(u, w)=1$ for all $w \in[u, v]$, and hence the value of the packing of $R\left(w^{\prime}\right)$ is $\varphi_{u}\left(w^{\prime}\right)$.

To compute these values we work through the vertices $v \in V$ moving in from the leaves to the root, and for $v$ fixed we first calculate $\varphi_{u}(v)$ for all $u \in V$ and then $H(v)$. After computing $H(v)$ we compute $x^{v}$, the associated optimal 1-packing of $R(v)$ having value $H(v)$. To calculate $x^{v}$, we set $x^{v}\left(u_{0}, v\right)=1$ if $H(v)=\operatorname{Max}_{u \in R(v)} \varphi_{u}(v)=$ $=\varphi_{u_{0}}(v)$ and trace back through $\varphi_{u_{0}}(v)$, and we set $x^{v}(z, v)=0$ for all $z \in V$ if $H(v)=$ $=\sum_{w \in S(v)} H(w)$ and then trace back through $H(w)$ for all $w \in S(v)$.

Theorem 3. $x=\sum_{v \in V} x^{v}(a(v)-a(p(v)))$ is an optimal solution to $\mathrm{P}_{2}$.

Proof. It is readily checked that $x$ belongs to $P_{2}$ and its value is $\sum_{v \in V} H(v)(a(v)-$ $-a(p(v)))$. 
Now we exhibit a dual feasible solution with the same value. The dual of $P_{2}$ is

$$
\begin{gathered}
\operatorname{Min} \sum_{v \in V} a(v) y(v) \\
y(v)+z(u, v)-\sum_{w \in Q(u, v)} z(u, w) \geqq c(u, v) \quad(u, v \in V, \quad u \neq v) \\
y(u)-\sum_{w \in Q(u)} z(u, w) \geqq c(u, u) \quad(u \in V) \\
y(v), \quad z(u, v) \geqq 0
\end{gathered}
$$

$\left(D_{2}\right)$

where $Q(u, v)$ is the set of vertices $w$ such that $v$ lies on the path from $u$ to $w$ and is a neighbour of $w$, and $Q(u)=Q(u, u)$ is the set of neighbours of $u$. In fact, we again construct the dual solution by a greedy algorithm. Set

$$
y(v)=H(v)-\sum_{w \in S(v)} H(w) .
$$

With this definition $P_{2}$ and $D_{2}$ have the same value. Now for each $u \in V$, ignore the initial ordering of the vertices, and consider a new ordering in which $u$ is the root. Working in from the leaves to the root $u$, define

$$
z(u, v)=\operatorname{Max}\left\{0, c(u, v)-y(v)+\sum_{w \in Q(u, v)} z(u, w)\right\} \text { for } v \in V .
$$

Clearly, $y, z \geqq 0$ and, by construction, the first inequalities of $\mathrm{D}_{2}$ are satisfied. To show that the second set is satisfied, consider the largest subtree $T \subseteq\{u\} \cup$ $\cup\{v \in V: z(u, v)>0\}$ containing $u$. Now

But

$$
c(u, u)-y(u)+\sum_{w \in Q(u)} z(u, w)=\sum_{w \in T}(c(u, w)-y(w)) .
$$

and hence

$$
H(r(T)) \geqq \sum_{v \in T} c(u, v)+\sum_{w \in S(T)} H(w)
$$

$$
\begin{aligned}
\sum_{v \in T}(c(u, v)-y(v)) & =\sum_{v \in T} c(u, v)-\sum_{w \in T} y(w) \\
& =\sum_{v \in T} c(u, v)-\left(H(r(T))-\sum_{w \in S(T)} H(w)\right) \leqq 0 .
\end{aligned}
$$

We mention here that essentially the same method works if in $\mathrm{P}_{2}$ one or more of the inequalities $\sum_{u \in V} x(u, v) \leqq a(v)$ is replaced by the equality $\sum_{u \in V} x(u, v)=$ $=a(v)$. If such a change occurs at a vertex $v \in V$, the recursion for $H$ just changes to

$$
H(v)=\operatorname{Max}_{\mu \in R(v)} \varphi_{u}(v)
$$




\section{Covering with a given family of subtrees} subtrees.

Now we consider the problem of finding a minimum value cover of $R$ by

$$
\begin{gathered}
Z_{0}=\operatorname{Min}\left\{\sum c(T) x(T): T \in \mathscr{F}\right\} \\
\sum\{x(T): T \ni v\} \geqq 1 \quad(v \in V) \\
x(T) \geqq 0 \text { and integer }(T \in \mathscr{F}) .
\end{gathered}
$$

We assume throughout that $c(T) \geqq 0$ as otherwise $Z_{0}=-\infty$ or $\mathrm{C}_{0}$ has no feasible solution.

We also consider two related problems:

$\left(\mathrm{C}_{1}\right)$ : Find a minimum value partition of $R$ using subtrees $S \subseteq T,(T \in \mathscr{F})$ where the cost $c(S, T)$ of each subtree $S \subseteq T$ is $c(T)$. In $C_{1}$ one tree $\bar{T} \in \mathscr{F}$ may contain several subtrees $S$ taking part in the partition. Let $Z_{1}$ be the optimal value of $C_{1}$. Formally

$$
\begin{gathered}
Z_{1}=\min \left\{\sum\{c(S, T) x(S, T): S \sqsubseteq T, T \in \mathscr{F}\} \text { subject to } x(S, T) \in\{0,1\}\right. \text { and } \\
\left.\sum\{x(S, T): v \in S, S \subseteq T, T \in \mathscr{F}\}=1 \text { for each } v \in V\right\} .
\end{gathered}
$$

$\left(\mathrm{C}_{2}\right)$ : Find a minimum value partition of $R$ using at most one subtree $S \subseteq T$ from each $T \in \mathscr{F}$ with cost $c(S, T)=c(T)$. Let $Z_{2}$ be the optimal value of $\mathrm{C}_{2}$. Formally

$$
\begin{gathered}
Z_{2}=\min \left\{\sum\{c(S, T) x(S, T): S \subseteq T, T \in \mathscr{F}\} \text { subject to } x(S, T) \in\{0,1\}\right. \text { and } \\
\sum\{x(S, T): v \in S, S \subseteq T, T \in \mathscr{F}\}=1 \text { for each } v \in V, \text { and } \\
\left.\sum\{x(S, T): S \subseteq T\} \leqq 1 \text { for each } T \in \mathscr{F}\right\} .
\end{gathered}
$$

Observation. $Z_{0} \leqq Z_{1} \leqq Z_{2}$.

We first show that there is a good algorithm for $C_{1}$ with the more general subtree cost function $c(S, T)=c(T)+\sum_{v \in S} c_{T, v}$ if $S \subseteq T$. In fact we reduce $C_{1}$ to the problem $P_{2}$.

$$
\text { Reduction of } \mathrm{C}_{1} \text { to } \mathrm{P}_{2}
$$

For each pair $(v, T)$ with $T \in \mathscr{F}$ and $v \in T$, add a dummy vertex $u_{v, T}$ to $R$, and an edge $\left(v, u_{v, T}\right)$ between the dummy vertex and $v$. On the resulting tree, define the values as follows:

$$
\begin{aligned}
c\left(u_{v, T}, u_{v, T}\right) & =-c(T) \quad(v \in T, T \in \mathscr{F}) \\
c\left(u_{v, T}, w\right) & =-c_{T, w} \quad(v \in T, T \in \mathscr{F}, w \in T) \\
c(u, v) & =-\infty \quad \text { otherwise. }
\end{aligned}
$$

The packing problem $\mathrm{P}_{2}$ on the extended graph with equalities $(=1)$ on the vertices of $R$ and inequalities ( $\leqq 1$ ) on the dummy nodes solves $C_{1}$. 
Now consider the solution of $\mathrm{C}_{2}$. We say that a set of subtrees $\mathscr{F}^{*} \sqsubseteq \mathscr{F}$ is a cover for $R$ if $U\left\{T: T \in \mathscr{F}^{*}\right\}=R$. A family $\mathscr{F}$ of subtrees is said to be partitionable if for every cover $\mathscr{F}^{*} \subseteq \mathscr{F}$ one can find a subtree $S(T) \subseteq T$ for each $T \in \mathscr{F}^{*}$ such that $\bigcup_{T \in \mathscr{F} *} S(T)=R$, and if $T_{1}, T_{2} \in \mathscr{F}^{*}$ with $T_{1} \neq T_{2}$ then $S\left(T_{1}\right) \cap$ $\cap S\left(T_{2}\right)=\emptyset$.

Proposition 4. $\mathrm{C}_{0}$ is well-solved for partitionable families, and $Z_{0}=Z_{1}=Z_{2}$.

Proof. As $\mathscr{F}$ is partitionable, every feasible solution of $\mathrm{C}_{0}$ gives rise to a solution of $\mathrm{C}_{2}$ of the same value and so $Z_{0}=Z_{1}=Z_{2}$. Hence to solve $\mathrm{C}_{0}$ it suffices to solve $\mathrm{C}_{1}$ and take as a cover $\mathscr{F}^{*}$ the set of trees $T \in \mathscr{F}$ used in the solution of $\mathrm{C}_{1}$.

Therefore we are interested in the existence of partitionable families.

Definition. Trees $T_{1}$ and $T_{2}$ of $R$ have a fork if there are vertices $x_{1}, y_{1} \in T_{1} \backslash T_{2}$ and $x_{2}, y_{2} \in T_{2} \backslash T_{1}$ such that $\left[x_{1}, y_{1}\right]$ and $\left[x_{2}, y_{2}\right]$ have a point in common. A family $\mathscr{F}$ of subtrees of $R$ is fork-free if no pair $T_{1}, T_{2} \in \mathscr{F}$ has a fork.

Figure 1 shows a tree $R$, and two subtrees with a fork.

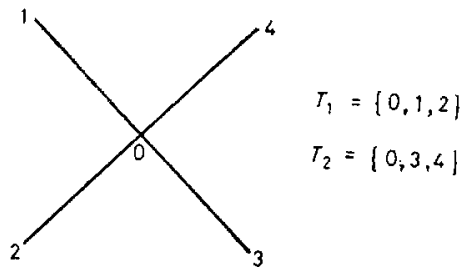

Fig. I. $T_{1}=\{0,1,2\}, T_{2}=\{0,3,4\}$

We will prove soon that a fork-tree family is partitionable. The converse is clearly not true. But given a tree $R$ and a family $\mathscr{F}$ of its subtrees and a fixed subtree $R^{\prime}$ of $R$, we say that $\mathscr{F}$ is partionable on $R^{\prime}$ if $\mathscr{F}^{\prime}=\left\{T \cap R^{\prime}: T \in \mathscr{F}\right\}$ is a partitionable family of subtrees of $R^{\prime}$. Then one can see easily that $\mathscr{F}$ is fork-tree if it is partitionable on all subtrees $R^{\prime}$ of $R$.

Theorem 5. There exists a "good" algorithm for the covering problem $\mathrm{C}_{0}$ when the family $\mathscr{F}$ of subtrees is fork-free.

Proof. It suffices to show that every fork-free family $\mathscr{F}$ is partitionable.

Consider a fork-tree family $\mathscr{F}$ that covers $R$ but no proper subfamily of $\mathscr{F}$ covers $R$. Consider then problem $C_{1}$ with each cost $c(S, T)=1$ for any $S \subseteq T \in \mathscr{F}$. We call a subtree $S$ of a tree $T \in \mathscr{F}$ a block if $x(S, T)=1$ in the given minimum value partition of $R$. In the given mininum value partition each block comes from a subtree $T \in \mathscr{F}$ and this subtree $T$ is thought to be fixed together with the block.

If the theorem is true then the value of $C_{1}$ is just $|\mathscr{F}|$. We argue by contradiction so we take a family $\mathscr{F}$ so that the number of blocks in the minimum value partition is larger than $|\mathscr{F}|$. For this $\mathscr{F}$, any minimum value partition contains two blocks $S^{1}(T)$ and $S^{2}(T)$ coming from the same tree $T \in \mathscr{F}$. Denote by $S^{1}\left(T_{0}\right)$ and $S^{2}\left(T_{0}\right)$ two such blocks whose distance apart is minimal. Consider now that mi- 
nimum value partition of $R$ for which this minimal distance (between $S^{1}\left(T_{0}\right)$ and $\left.S^{2}\left(T_{0}\right)\right)$ is minimum.

This minimum distance is realized by a path (in $R$ or in $T_{0}$ ) $P=\left\{v_{0}, v_{1}, \ldots, v_{n}\right\}$ where $v_{0} \in S^{1}\left(T_{0}\right), v_{n} \in S^{2}\left(T_{0}\right)$. Clearly $n>1$ as otherwise the block $S^{1}\left(T_{0}\right) \cup S^{2}\left(T_{0}\right)$ could replace the two blocks $S^{1}\left(T_{0}\right), S^{2}\left(T_{0}\right)$ and the value of this partition would be less.

The point $v_{1}$ is covered by a block $S\left(T_{1}\right)$ coming from some $T_{1} \in \mathscr{F} . T_{1}$ is different from $T_{0}$ for otherwise the distance between $S\left(T_{1}\right)$ and $S^{2}\left(T_{0}\right)$ would be less than that between $S^{1}\left(T_{0}\right)$ and $S^{2}\left(T_{0}\right)$.

Claim 1. $S^{1}\left(T_{0}\right) \Phi T_{1}, S^{2}\left(T_{0}\right) \nsubseteq T_{1}, S\left(T_{1}\right) \nsubseteq T_{0}$.

Proof. If, for instance, $S^{1}\left(T_{0}\right) \leqq T_{1}$, then $S^{1}\left(T_{0}\right) \cup S\left(T_{1}\right)$ would be a block of $T_{1}$ in a partition of $R$ with smaller value. The other two cases are similar and left to the reader.

Denote now by $B^{1}, \ldots, B^{p}$ the branches of $S\left(T_{1}\right)$ that stem from $v_{1}$ but do not contain $v_{2}$ and set $B^{0}=S\left(T_{1}\right) \backslash\left(\left\{v_{1}\right\} \cup B^{1} \cup \ldots \cup B^{p}\right)$. ( $B_{0}$ may be empty.) Clearly $v_{1} \in T_{0}$ and by Claim $1, B^{i} \subseteq T_{0}$ cannot hold for every $i=0,1, \ldots, p$.

Claim 2. There is exactly one $i \in\{0, \ldots, p\}$ with $B^{i} \Phi T_{0}$.

Proof. Assume, on the contrary, that $B^{i} \nsubseteq T_{0}$ and $B^{j} \nsubseteq T_{0}$ and choose a point $x_{1} \in B^{\backslash} \backslash T_{0}, x_{2} \in B^{j} \backslash T_{0}$. By Claim 1 there are points $y_{1} \in S^{1}\left(T_{0}\right) \backslash T_{1}$ and $y_{2} \in$ $\in S^{2}\left(T_{0}\right) \backslash T_{0}$. Then the two trees $T_{0}, T_{1} \in \mathscr{F}$ have a fork because the paths $\left[x_{1}, x_{2}\right]$ and $\left[y_{1}, y_{2}\right]$ meet at $v_{1}$.

Now we are going to construct two new blocks from $S^{1}\left(T_{0}\right) \cup S\left(T_{1}\right)$ which will form (together with the other blocks) a minimum value partition of $R$ with minimal distance less than $n$. Let $B^{i}$ be the unique branch with $B^{i} \varsubsetneqq T_{0}$. Then $\left(S^{1}\left(T_{0}\right) \cup S\left(T_{1}\right)\right) \backslash B^{i}$ and $B^{i}$ are two new blocks, the first coming from $T_{0}$ and the second from $T_{1}$. The distance from $S^{2}\left(T_{0}\right)$ to the new block $\left(S^{1}\left(T_{0}\right) \cup S\left(T_{1}\right)\right) \backslash B^{i}$ is less than $n$. This is a contradiction.

\section{Further observations}

A 0 -1 matrix $A$ is called a tree-matrix if it is the node versus subtree incidence matrix of a subtree family of a tree. A clique in a graph is the vertex set of a maximal complete subgraph.

Proposition 6. $A$ is a tree-matrix if and only if $A$ is the clique-node incidence matrix of a triangulated graph, including possibly some dominated rows.

This is easily proved using the Helly property of the subtrees of a tree and a theorem of Gavril [3] saying that the intersection graphs of subtrees of a tree are exactly the triangulated graphs. The proof is left to the reader.

It follows that we can consider the primal and dual variables for $P_{1}$ as node packing and clique weights in the corresponding triangulated graphs. If one checks now how our algorithm for $\mathrm{P}_{1}$ works on the corresponding triangulated graph, 
one can see that it does essentially the same thing as a perfect elimination scheme (see Golumbic [4]). In this sense what is new in Theorem 1 is the replacement of $a(v) \equiv 1$ by a monotone function.

As triangulated graphs can be recognised by a polynomial time algorithm it is of no surprise that the same is true for tree-matrices.

\section{An Algorithm to Recognise Subtree Matrices}

Let $M$ be a 0-1 matrix

a) If $M$ has a unit column $e_{j}$, set $M-M \backslash e_{j}$. row $t$ from $M$ )

b) If $M$ has no unit columns and $m_{t j} \leqq m_{s j}$ for all $j$, set $M \leftarrow M \backslash m_{t}$ (drop tree matrix.

c) If $M$ has no unit columns and the rows form a clutter, stop. $M$ is not a

d) Repeat steps a), b), c) until $M=0$, then $M$ is a tree matrix.

The validation of the algorithm is left to the reader, we only prove c): any tree representation of $M$ must have a leaf $t$. Either there exists a tree $T=\{t\}$ giving rise to a unit column or every tree containing $t$ contains its neighbour $s$. In other words $m_{s j} \geqq m_{t j}$ for all $j$ where $s$ is the neighbour of $t$.

We now consider the covering problem $C_{0}$. In general it is NP-hard as any $0-1$ covering problem reduces to it by adding a row of l's. Therefore it is not surprising that its linear programming relaxation does not in general have a $0-1$ solution. The simplest example showing this is the tree $R$ depicted in Figure 2.

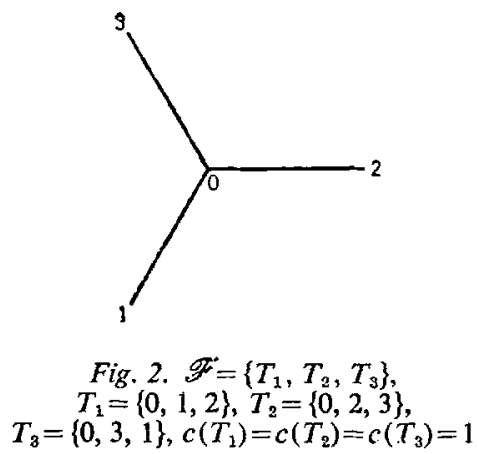

Given that $\mathrm{C}_{0}$ is well-solved for fork-free families, one might ask whether in such cases the linear programming relaxation has a $0-1$ solution. But the three subtrees appearing in Figure 2 are fork-free, so this is not the case. However it is known that the associated linear program has an integer solution when dealing with distance subtrees $T_{u}=\{v \in V: d(v, u) \leqq \alpha\}$, and more generally when the $0-1$ incidence matrix of the subtrees is totally balanced (see Kolen [7], Hoffman, Kolen, Sakarovitch [6] for details). It is readily shown using for instance the above algorithm that every totally balanced matrix is a subtree matrix.

Now both totally balanced (TB) matrices and fork-free (FF) families give rise to subtree matrices (SM). Are (TB) and (FF) related? The example of Figure 2 
is forkfree, but cannot be totally balanced as the LP relaxation is noninteger. On the other hand, there exists a totally balanced matrix, for which there exists a unique representation as a family of subtrees and the family is not forkfree. Finally it is readily checked that the distance subtrees defined above are forkfree, so FF $\cap \mathrm{TB} \neq \emptyset$.

Finally we return to problem $P_{2}$. We consider the econonic lot sizing problem with backlogging, which was one of the problems motivating this research. The problem can be formulated as a simple plant location problem where the plants are located on an interval, (or in the terminology of this paper: at the vertices of a tree $R$ having vertex set $V=\{1, \ldots, n\}$ and edges $(i, i+1), i=1, \ldots, n-1$, so the tree is a simple path).

The formulation is:

$$
\begin{gathered}
\text { Min } \sum_{u=1}^{n} \sum_{v=1}^{n} c(u, v) x(u, v) \\
\sum_{u=1}^{n} x(u, v)=1 \quad(v \in\{1, \ldots, n\}) \\
x(u, v)-x(u, u) \leqq 0 \quad(u, v \in\{1, \ldots, n\}, \quad u \neq v) \\
x(u, v) \geqq 0 \quad(u, v \in\{1, \ldots, n\}),
\end{gathered}
$$

where $x(u, v)$ is the fraction of the demand in period $v$ produced in period $u, c(u, u)$ is the fixed cost of setting up in period $u$ plus $\left.p_{u} d_{u}, c(u, v)=p_{u}+c_{u}^{+}+\ldots+c_{v-1}^{+}\right) d_{v}$ if $u>v, c(u, v)=\left(p_{u}+c_{u}^{-}+\ldots+c_{v+1}^{-}\right) d_{v}$ if $v<u$, where $p_{u}$ is the price of unit production in period $u, c_{u}^{+} \geqq 0$ and $c_{u}^{-} \geqq 0$ are the price of unit transportation from $u$ to $u+1$ and $u+1$ to $u$ respectively and $d_{v}$ is the demand in period $v$. It is typically imposed that $x(u, u) \in\{0,1\}$. Now we can show

Theorem 7. ELS has an optimal 0-1 solution.

Outline of proof. It is easily shown that optimal solutions to ELS satisfy the inequalities

and

$$
x(u, u) \geqq x(u, u+1) \geqq x(u, u+1) \geqq \ldots
$$

$$
x(u, u) \geqq x(u, u-1) \geqq x(u, u-2) \geqq \ldots, \text { for all } u \in\{1, \ldots, n\} .
$$

Adding these inequalities to ELS, and removing the dominated inequalities, the resulting linear program is of the form $P_{2}$, with $R$ an interval graph, $a(v)=1$ for $v \in V$, and equality constraints at each node. It follows from Theorem 3 that ELS has an optimal integer solution. 


\section{References}

[1] I. BáránY, T. J. VAN RoY, and L. A. WolseY, Uncapacitated Lot-Sizing: The Convex Hullof Solutions, Mathematical Programming Study, 22 (1984), 32-43.

[2] A. Frank, Some Polynomial Algorithms for Certain Graphs and Hypergraphs, Proceedings of the 5th British Combinatorial Conference, Congressus Numerantium XV, Utilitas Math., Winnipeg (1976).

[3] F. GavRIL, The intersection Graphs of Subtrees in Trees are Exactly the Chordal Graphs, Journal of Combinatorial Theory, $B$ 16, (1974), 47-56.

[4] M. C. Golumbic, Algorithmic Graph Theory and Perfect Graphs, Academic Press, (1980).

[5] H. Gröflin and T. M. Liebling, Connected and Alternating Vectors: Polyhedra and Algorithms, Mathematical Programming, 20 (1981), 233-244.

[6] A. J. Hoffman, A. W. J. Kolen and M. Sakarovitch, Totally Balanced and Greedy Matrices, Prepring BW 165/82, Stiching Mathematisch Centrum, Amsterdam, (1982).

[7] A. W. J. KoLeN, Solving Covering Problems and the Uncapacitated Plant Location Problem on Trees, Preprint BW 163/82, Stichting Mathematisch Centrum, Amsterdam (1982).

I. Bárány

Mathematical Institute of the Hungarian Academy of Sciences Redilanoda u. 13-15. 1053, Hungary
L. A. Wolsey

Center Op. Res, Econometrics Université Catholique de Louvain 1348 Louvain-la-Neuve Belgium

\section{J. Edmonds}

Dept. of Combinatorics and Optimization University of Waterloo Ontario, Canada 